\title{
Влияние рентгеновского излучения на оптические свойства фоторефрактивных кристаллов силиката висмута
}

\author{
(C) В.Т. Аванесян, И.В. Писковатскова, В.М. Стожаров \\ Российский государственный педагогический университет им. А.И. Герцена, \\ 191186 Санкт-Петербург, Россия \\ E-mail: avanesyan@mail.ru
}

Поступила в Редакцию 26 марта 2019 г.

В окончательной редакции 5 апреля 2019 г.

Принята к публикации 8 апреля 2019 г.

\begin{abstract}
Представлены результаты исследования спектров оптического поглощения в монокристаллах силиката висмута $\mathrm{Bi}_{12} \mathrm{SiO}_{20}$. Определены ширина запрещенной зоны и характеристическая энергия Урбаха. Установлено влияние предварительного рентгеновского облучения на поведение экспериментальных спектральных зависимостей и значения характеристических параметров, обусловленное дефектной структурой силиката висмута.
\end{abstract}

Ключевые слова: силикат висмута, силленит, спектральная зависимость, оптическое поглощение, рентгеновское излучение.

DOI: 10.21883/FTP.2019.08.47992.9115

\section{1. Введение}

Интерес к нецентросимметричным кубическим кристаллам силиката висмута $\mathrm{Bi}_{12} \mathrm{SiO}_{20}(\mathrm{BSO})$ связан с их высокой чувствительностью к оптическому излучению, которая проявляется в фотопроводимости, фоторефрактивном и фотохромном эффектах [1]. Благодаря уникальному сочетанию величины и скорости фотоотклика, a также возможности изготовления кристаллических образцов различной ориентации с необходимыми размерами указанные материалы силленитной группы типа $\mathrm{Bi}_{12} \mathrm{MO}_{20}(\mathrm{M}-\mathrm{Si}, \mathrm{Ge}, \mathrm{Ti})$ широко используются в динамической голографии [2,3]. На процесс поглощения электромагнитного излучения кристаллом значительное влияние оказывает существование структурных дефектных центров, приводящее к формированию локальных энергетических уровней в запрещенной зоне и обусловливающее особенности оптических, электрических и других свойств нелегированных кристаллов силленитов. Присутствием локальных состояний, в частности, объясняется наличие у указанных материалов фоторефрактивных и фотохромных свойств.

Одним из методов повышения фоторефрактивной чувствительности кристаллов силленитной группы является рентгеновское облучение исследуемых образцов [4]. Воздействие рентгеновского излучения может приводить к нарушению стехиометрии, к изменениям в распределении и структуре дефектов в кристаллах, что в свою очередь оказывает существенное влияние на характер оптического поглощения материала. К настоящему времени проблемы, связанные с природой и энергетическими параметрами центров в кристаллах силленитной структуры, ответственных за оптические свойства в области края фундаментального поглощения, не получили решения.
В настоящей работе исследовалось влияние предварительного рентгеновского облучения на оптические характеристики образцов фоторефрактивных кристаллов BSO при различном времени облучения.

\section{2. Эксперимент}

Образцами для исследования являлись кристаллы, выращенные методом Чохральского, которые представляли собой оптически полированные пластины желтого цвета толщиной $d=0.75-1$ мм. При использовании однолучевого спектрофотометра СФ-56 регистрировались спектры оптической плотности монокристаллов с шагом 1 нм в диапазоне длин волн $\lambda=200-1000$ нм. С применением рентгеновского дифрактометра ДРОН-7 проводилось предварительное облучение образцов кристаллов жестким излучением на линии $\mathrm{Cu} K_{\alpha 1}$ при значении энергии рентгеновского кванта, равном 8.047 эВ. Все измерения проводились при температуре $T=293 \mathrm{~K}$.

\section{3. Результаты и обсуждение}

На рис. 1 приведены спектральные зависимости оптической плотности $D(\lambda)$ исследуемых кристаллов BSO, полученные до и после процесса рентгеновского облучения в течение 30 и 90 мин соответственно. В спектрах $D(\lambda)$ можно выделить два участка: фундаментального и слабого поглощения, на котором функция имеет экспоненциальный характер. Зависимость, полученная без предварительного рентгеновского облучения, характеризуется резким уменьшением значения оптической плотности $D$ в области длин волн $400<\lambda<500$ нм. Как следует из полученных данных, максимальная оптическая плотность в исследуемых кристаллах наблюдается в ультрафиолетовой области спектра. 


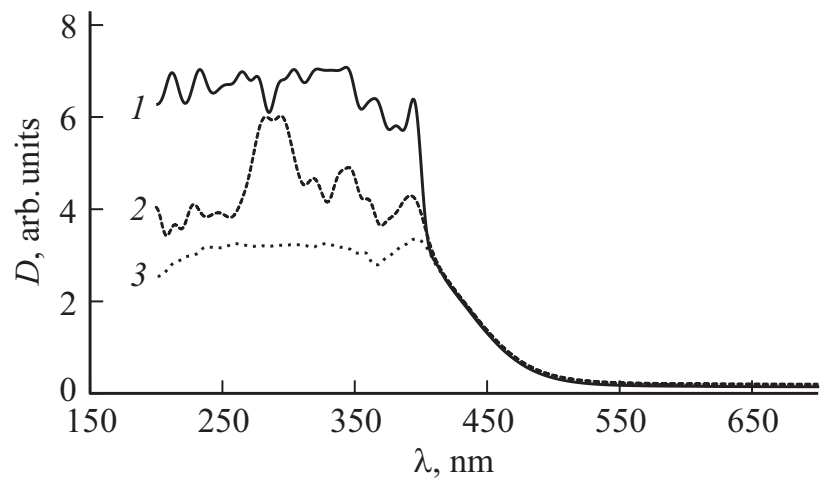

Рис. 1. Спектральная зависимость оптической плотности $D(\lambda)$ кристаллов BSO: 1 - в отсутствие рентгеновского облучения; 2,3 - при облучении в течение 30 и 90 мин соответственно.

При воздействии рентгеновского излучения на исследуемый кристалл имеет место уменьшение параметра $D$ в области, предшествующей краю фундаментального поглощения. Увеличение времени воздействия рентгеновского излучения приводит к снижению оптической плотности.

С учетом известных соотношений связь между коэффициентом поглощения $\alpha$ и оптической плотностью $D$ определяется выражением

$$
\alpha=2.3 D / d,
$$

где $d-$ толщина образца кристалла.

Для определения ширины запрещенной зоны обычно используется область фундаментального поглощения, соответствующая электронным переходам зона-зона, в которой спектральная зависимость коэффициента поглощения определяется формулой Тауца [5]

$$
(\alpha h v)^{1 / m}=A\left(h v-E_{g}\right),
$$

$\alpha-$ коэффициент поглощения, $h-$ энергия падающих фотонов ( $v$ - частота оптического излучения), $A-$ постоянная, зависящая от природы оптических переходов, $E_{g}$ - оптическая ширина запрещенной зоны, $m$ определяется типом перехода, зависящим от структуры материала. Для прямозонной структуры исследуемого полупроводника доминирующими должны быть переходы, соответствующие $m=1 / 2$.

Таким образом, спектральная зависимость коэффициента поглощения для прямых разрешенных переходов описывается формулой

$$
\alpha=\left[A\left(h v-E_{g}\right)\right]^{1 / 2} / h v .
$$

Экстраполяция линейного участка спектра края поглощения в виде функции $\alpha^{2}(h v)$ к оси $h v$ (рис. 2) позволяет найти значение оптической ширины запрещенной зоны полупроводника как длину отрезка, отсекаемого на оси энергий. Предварительное воздействие рентгеновского излучения в течение 30 мин приводит к уменьшению оптической ширины запрещенной зоны с 3.05 до 2.95 эВ. При времени облучения 90 мин значение $E_{g}$ уменьшилось до 2.85 эВ.

Область энергий $E_{g}$, в которой возможно поглощение оптического излучения, получила название „хвоста“ Урбаха (Urbach tail). На этом участке зависимость $\alpha(h v)$ отвечает экспоненциальному характеру изменения и подчиняется правилу [6]

$$
\alpha(v)=\alpha_{0} \exp \left[\left(h v-E_{g}\right) / E_{U}\right]
$$

где $\alpha_{0}-$ константа, $E_{U}-$ так называемая характеристическая энергия Урбаха, которая определяется как отсечка при экстраполяции линейной части зависимости $\ln (\alpha)=f(h v)$ (рис. 3) на ось энергий. Формирование „урбаховского хвоста“, в частности в кристаллических материалах, может быть обусловлено наличием разупорядочения в ближнем порядке при флуктуации значений постоянной решетки и углов между связями $[7,8]$.

Найденное значение параметра $E_{U}$ после предварительного рентгеновского облучения в течение 30 мин уменьшилось с 2.25 до 2.15 эВ и осталось неизменным при увеличении времени облучения до 90 мин.

Изучение спектральных зависимостей оптического поглощения полупроводника, кристаллическая решетка

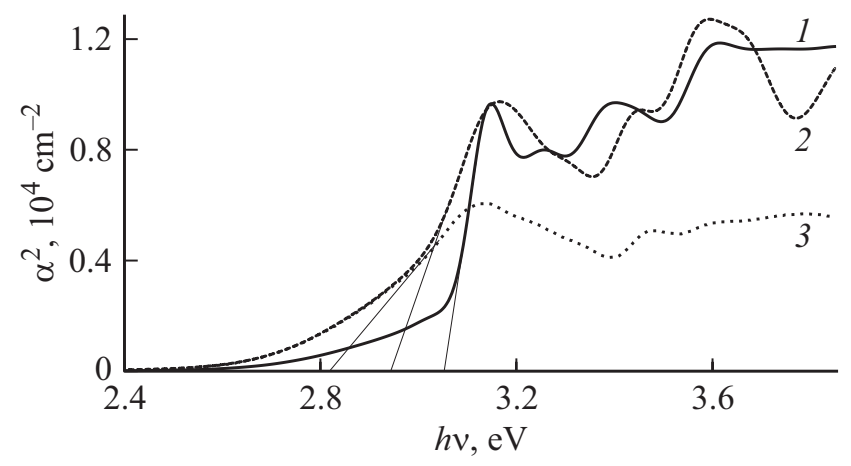

Рис. 2. Зависимость $\alpha^{2}(h v)$ кристаллов BSO: $1-$ в отсутствие рентгеновского облучения; 2,3 - при облучении в течение 30 и 90 мин соответственно.

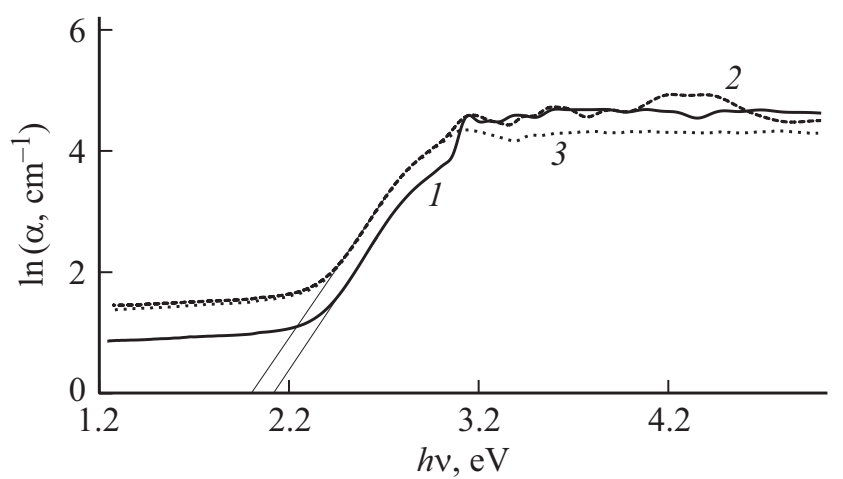

Рис. 3. Зависимость $\ln \alpha(h v)$ кристаллов BSO: 1 - в отсутствие рентгеновского облучения; 2,3 - при облучении в течение 30 и 90 мин соответственно. 


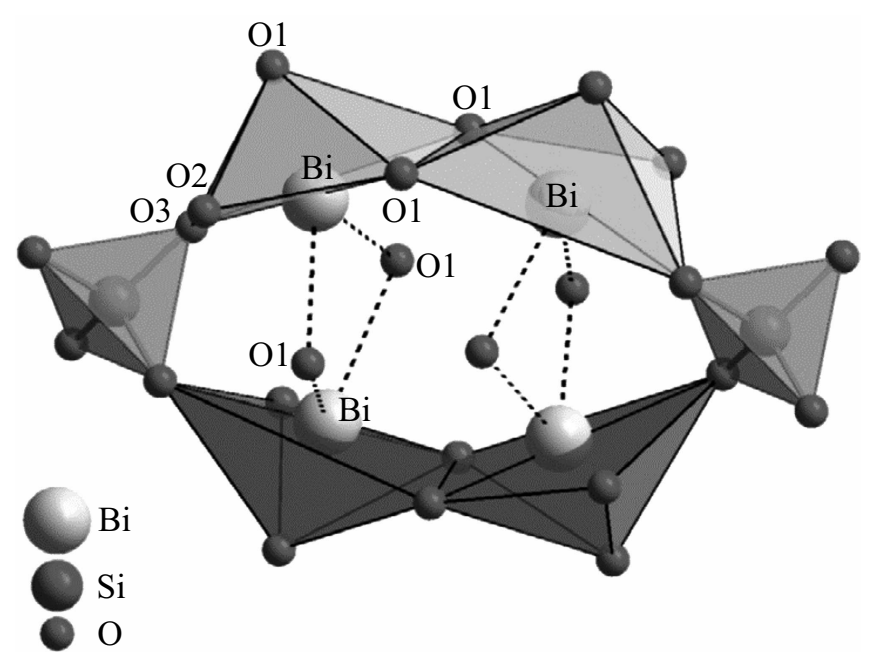

Pис. 4. Образование полости в структуре BSO с локализованными неподеленными электронными парами при объединении октаэдра $\mathrm{BiO}_{5}$ и тетраэдра $\mathrm{SiO}_{4}[11]$.

которого включает структурные дефекты, является одним из эффективных методов, позволяющих установить корреляцию между особенностями строения и оптическими свойствами кристалла. Энергию $E_{U}$, как известно, определяет поглощение электронами на ловушках в запрещенной зоне, появление которых вызвано присутствием структурных дефектов, формирующих „хвосты“ валентной зоны и зоны проводимости и оказывающих влияние на распределение потенциала кристаллической решетки.

Формирование собственных дефектных центров, преобладающих в кристаллической решетке BSO, связано с изоморфизмом ионов $\mathrm{Bi}^{3+}$ и $\mathrm{Si}^{4+}$ в тетраэдрах $\mathrm{SiO}_{4}$ [9] (рис. 4), в котором принимает участие стереохимически активная электронная одиночная пара $6 s^{2}$ иона $\mathrm{Bi}^{3+}[10]$. Эти пары принадлежат комплексам $\mathrm{BiO}_{7}$ и расположены в полостях, образованных четырьмя пирамидами $\mathrm{BiO}_{5}$ неправильной формы и двумя правильными тетраэдрами $\mathrm{SiO}_{4}$. Антиструктурные ионы $\left(\mathrm{Bi}_{\mathrm{Si}}^{3+}\right)$ и $\left(\mathrm{Bi}_{\mathrm{Si}}^{5+}\right)$ замещают ионы $\mathrm{Si}^{4+}$ в кислородных тетраэдрах, образуя дефекты $\mathrm{BiSiO}_{4}$ и $\mathrm{BiSiO}_{3}$ соответственно. Указанные группы дефектов обусловливают наличие в кристаллах силленитов спектра локальных состояний вблизи уровня Ферми, по которым осуществляется перенос (перезарядка) носителей заряда в процессе оптического возбуждения.

В процессе рентгеновского облучения исследуемых кристаллов вероятным является искажение октаэдров $\mathrm{BiO}_{5}$, полиэдров $\mathrm{BiO}_{7}$ и тетраэдров $\mathrm{SiO}_{4}$, что приводит к формированию дополнительного числа глубоких и мелких локальных состояний в запрещенной зоне. Возникновение метастабильных радиационных локальных центров в процессе рентгеновского облучения и последующая их оптическая перезарядка под действием вторичных электронов вызывают локальные изменения краев энергетических зон полупроводника и соответственно флуктуации ширины запрещенной зоны [11].

В процессе расшифровки данных, полученных с помощью дифрактометра ДРОН-7, было установлено блочное строение, полиморфизм кубической структуры исследуемого силленита и некоторое изменение постоянной решетки после предварительной рентгеновской обработки образцов. Средний размер блоков рассчитывался с применением формулы Селякова-Шеррера [12]:

$$
d=K \lambda /(\beta \cos \theta),
$$

где $K$ - безразмерный коэффициент формы частиц (постоянная Шеррера), $\lambda$ - длина волны рентгеновского излучения, $\beta$ - ширина рефлекса на полувысоте пика и $\theta-$ угол дифракции рентгеновских лучей. Зависимость размера блоков $d$ от длительности облучения образцов представлена на рис. 5 (кривая 1).

Наличие структурных элементов (блоков) предполагает вероятность существования краевых дислокаций различного типа за счет возникновения значительных механических напряжений и, таким образом, представляет собой дополнительный источник дефектообразования в кристалле BSO. Плотность дислокаций, т.е. число линий дислокаций, пересекающих единичную площадку в кристалле, расположенную под определенным углом, является тензором второго ранга и для кубической решетки может быть рассчитана по формуле [13]

$$
\rho=\left[3 \eta g \varepsilon^{2} /\left(d^{2} b^{2} F\right)\right]^{1 / 2},
$$

где $\eta$ - фактор формы, составляющий для дифрактометра ДРОН-7 значение $\eta=0.6, \varepsilon-$ относительная деформация, $b=a / \sqrt{2}-$ вектор Бюргерса ( $a-$ постоянная решетки), $g$ - коэффициент, зависящий от формы распределения, описывающего профиль пика и рассчитываемый с применением программного обеспечения установки ДРОН-7, $F-$ параметр взаимодействия между дислокациями, $n$ - число дислокаций в блоке (при отсутствии полигонизации $F=n$ ).

Длительность рентгеновского облучения может оказывать существенное влияние также на плотность дислокаций (рис. 5, кривая 2). При этом перестройка блоков,

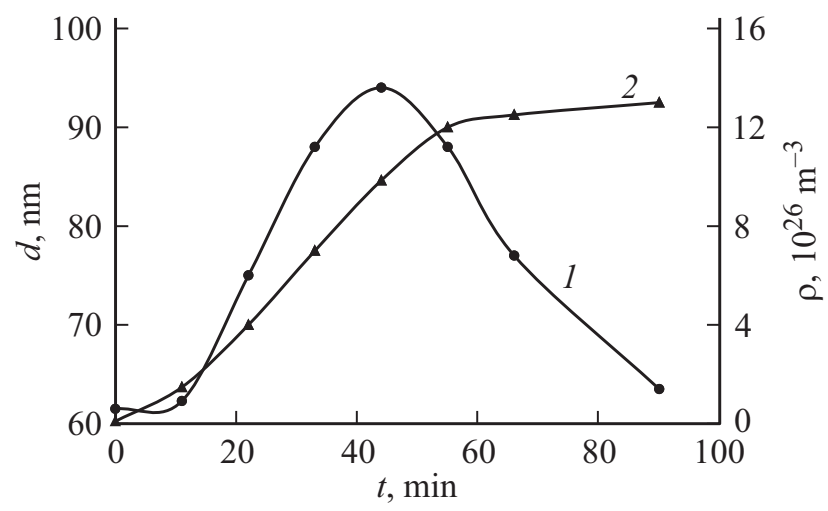

Рис. 5. Зависимость размера блоков (1) и плотности дислокаций (2) от времени рентгеновского облучения кристаллов BSO. 
которые отличаются в выбранном направлении своим значением межплоскостного расстояния, в процессе облучения может приводить как к их слиянию для малых времен облучения, так и к дроблению при повышенной плотности дислокаций с увеличением значения указанного времени.

\section{4. Заключение}

На основе экспериментальных данных получены спектральные зависимости оптического поглощения кристаллов силиката висмута. Анализ экспериментальных результатов позволил определить ширину запрещенной зоны и характеристическую энергию Урбаха. Установлено влияние длительности процесса предварительного рентгеновского облучения исследуемых образцов кристаллов BSO на характер поведения спектральных кривых и значения энергетических параметров. Комплексные ионы висмута $\mathrm{BiO}_{7}$, связанные с вакансиями кремния, являются одним из основных структурных образований силиката висмута, обусловливающих оптическое поглощение вблизи края фундаментального поглощения в видимой области спектра. В процессе рентгеноструктурного анализа установлен дополнительный источник дефектообразования, а именно изменение геометрии блочной структуры образца $\mathrm{Bi}_{12} \mathrm{SiO}_{20}$ и плотности дислокаций в процессе рентгеновского облучения, что в свою очередь может приводить к варьированию значения характеристической энергии Урбаха.

\section{Конфликт интересов}

Авторы заявляют, что у них нет конфликта интересов.

\section{Список литературы}

[1] В.К. Малиновский, О.А. Гудаев, В.А. Гусев, С.И. Деменко. Фотоиндуцированные явления в силленитах (Новосибирск, Наука, 1990).

[2] М.П. Петров, С.И. Степанов, А.В. Хоменко. Фоторефрактивные кристаллы в когерентной оптике (СПб., Наука, 1992).

[3] M.G. Kisteneva, A.S. Akrestina, S.M. Shandarov, S.V. Smirnov. J. Holography Speckle, 5, 280 (2009).

[4] Ig. Dementiev, E. Maximov, E. Pocotilov, L. Tarakanova. J. Moldavian J. Phys. Sci., 4 (1), 54 (2005).

[5] J. Tauc, R. Grigorovici, A. Vancu. Phys. Status Solidi, 15, 627 (1966).

[6] F. Urbach. Phys. Rev., 92, 1324 (1953).

[7] Г.Я. Клява. ФТТ, 27 (5), 1350 (1985).

[8] П.Г. Петросян, Л.Н. Григорян. ЖТФ, 87 (3), 443 (2017).

[9] В.Т. Аванесян, К.И. Пайма, В.М. Стожаров. ФТТ, 59 (6), 1056 (2017).

[10] В.Т. Аванесян, Н.М. Абрамова. ФТТ, 57 (6), 1084 (2015).

[11] L. Wiehl, A. Friedrich, E. Haussühl, W. Morgenroth, A. Grzechnik, K. Friese, B. Winkler, K. Refson, V. Milman. J. Phys.: Condens. Matter, 22, 505401 (2010).
[12] В.Б. Вайнштейн, Л.М. Инденбом, В.М. Фридкин. Современная кристаллография (М., Наука, 1979).

[13] C.K. De, N.K. Mishra. Indian J. Phys., 71, 530 (1997).

Редактор Л.В. Шаронова

\section{Effect of $X$-ray radiation on optical properties of bismuth silicate photorefractive crystals}

\section{V.T. Avanesyan, I.V. Piskovatskova, V.M. Stozharov \\ Herzen State Pedagogical University of Russia, 191186 St. Petersburg, Russia}

Abstract The results of the study of optical absorption spectra bismuth silicate $\left(\mathrm{Bi}_{12} \mathrm{SiO}_{20}\right)$ in single crystals are presented. The energy gap width and the characteristic Urbach energy are determined. The effect of preliminary $x$-ray on the behavior of the experimental spectral dependences and the characteristic parameters values due to the bismuth silicate defect structure is established. 